Rev. Bras. Saúde Prod. Anim., Salvador, v.17, n.4, p.685-695 out./dez., 2016 http://www.rbspa.ufba.br

\title{
Desempenho, características da carcaça e qualidade da carne de novilhos cruzados Hereford-Angus alimentados com silagem de grão úmido de sorgo
}

\author{
Carcass and meat quality traits of crossbred Angus-Hereford calfs fed with Sorghum \\ grain silage
}

\author{
CHIAIA, Hermenegildo Lucas Justino ${ }^{1 *}$; BALDI, Fernando ${ }^{1}$; PEREIRA, Angélica \\ Simone $\mathrm{Cravo}^{2}$; BANCHERO, Georgget ${ }^{3}$; BRITO, Gustavo ${ }^{3}$; LA MANNA, Alejandro ${ }^{3}$; \\ FERNANDEZ, Enrique ${ }^{3}$; ACOSTA, Yamandú ${ }^{3}$; MONTOSSI, Fabio ${ }^{3}$; GASALLA, \\ Pablo $^{4}$; MIGLIERINA, Agustín Fernández ${ }^{4}$; NUÑEZ, Agustin ${ }^{4}$
}

\footnotetext{
${ }^{1}$ Universidade Estadual Paulista, Faculdade de Ciências Agrárias e Veterinárias, Departamento de Zootecnia, Jaboticabal, São Paulo, Brasil.

${ }^{2}$ Universidade de São Paulo, Departamento de Nutrição e Produção Animal, Pirassununga, São Paulo, Brasil

${ }^{3}$ Instituto Nacional de Investigación Agropecuaria, Colonia, Uruguay

${ }^{4}$ Universidad de la República, Montevideo, Facultad de Agronomía, Uruguay

*Endereço para correspondência: chiaia1@yahoo.com.br
}

\section{RESUMO}

Objetivou-se avaliar três tipos de silagem de grão úmido de sorgo (alto tanino, baixo tanino e branco), na forma de silagem de grão úmido, combinados com duas formas de fornecimento da ração (volumoso e concentrado fornecidos de forma separada ou misturada), sobre o consumo de matéria seca, evolução do peso vivo, ganho de peso médio diário, conversão alimentar e principais características de carcaça (área de olho de lombo, espessura de gordura subcutânea e marmorização in vivo e post mortem) e carne (cor, maciez e pH) de novilhos cruzados Hereford-Angus. Foram utilizados 48 bovinos machos, castrados, cruzados Hereford-Angus, com idade média de 20 meses, divididos em blocos casualizados, em fatorial $3 \times 2$. A regressão do ganho de peso de cada tratamento foi comparada utilizando-se contrastes ortogonais. Para consumo de matéria seca relativo, houve efeito do tipo de sorgo $(\mathrm{P}=0,002)$ e forma de fornecimento da ração $(\mathrm{P}=0,0005)$. A forma de fornecimento da ração pode interferir sobre o consumo de matéria seca absoluto e peso vivo médio $(\mathrm{P}=0,0001)$. Para a conversão alimentar foi observado efeito do tipo de sorgo $(\mathrm{P}=0,04$. Observou-se efeito da forma de fornecimento da ração para a marmorização $(\mathrm{P}=0,04)$ post mortem. Não houve efeito do tipo de sorgo e da forma de fornecimento da ração sobre as características de carcaça e pesos dos cortes cárneos. O fornecimento do volumoso separado do concentrado na alimentação de bovinos confinados é viável do ponto de vista operacional e nutricional.

Palavras-chave: bovinos de corte, crescimento, carcaça, qualidade da carne, tanino

\section{SUMMARY}

This work aimed to evaluate three varieties of sorghum as wet grain silage (high, low and white tannin), combined with two form of food supply (concentrated with roughage separated or mixed) on dry matter intake, body weight evolution, average daily growth, feed conversion, main meat and carcass traits (rib eye area, fat thickness and marbling in vivo and post mortem) in Hereford-Angus crossbred steers. Forty eight males castrated with 20 months of age were used, divided into randomized blocks, arranged in a $3 \times 2$ factorial. The regression coefficients for the weight gain of each treatment was compared using orthogonal contrasts. Dry matter intake was affected by the type of sorghum $(\mathrm{P}=0.002)$ and the form of food supply ( $\mathrm{P}=0.0005)$. The form of food supply can interfere with the dry matter intake $(\mathrm{P}=0.0001)$ and weight $(\mathrm{P}=0.0001)$. Feed 
conversion and marbling, was affected by the type of sorghum $(\mathrm{P}=0.04)$. Meat marbling measured at deboning was affected by the form of food supply $(\mathrm{P}=0.04)$. The type of sorghum and form of food supply did not influence carcass characteristics and weights of meat cuts. The concentrated with roughage separated supply is nutritionally and operationally preferable.

Keywords: beef cattle, growth traits, carcass traits, meat quality, tannin

\section{INTRODUÇÃO}

O cultivo de sorgo (Sorghum bicolor) apresenta algumas vantagens como a menor exigência de nutrientes, maior adaptabilidade ao solo e ao ambiente e baixo preço de instalação e manejo do cultivo. Porém, o amido presente no grão de sorgo é menos degradável no rúmem, pela presença de tanino (ROONEY \& PFLUGFELDER, 1986). Atualmente no mercado, encontra-se disponível uma ampla variedade de sorgos, com o objetivo de produção de grãos com diferentes tipos de pigmentação, como sorgos alto tanino, baixo tanino e branco, porém, existe pouca informação em relação à resposta animal obtida com a sua utilização na forma de silagem.

Em dietas com alta proporção de concentrados, o volumoso além de proporcionar nutrientes para o animal é fonte de fibra, mantém a atividade normal da mastigação, funcionamento do rúmen para a digestibilidade e absorção dos nutrientes. Portanto, a falta de fibra na alimentação dos bovinos pode causar queda na produção e problemas de saúde que podem ser causa de morte.

A forma de fornecimento do volumoso, em dietas com alto nível de concentrado, para ruminantes é um aspecto importante do ponto de vista operacional e nutricional. Neste sentido, Atwood et al. (2001), estudaram o efeito de diferentes formas de fornecimento da ração (volumoso e concentrado fornecidos de forma conjunta ou separada). Da mesma forma, La Manna et al. (2012) estudaram o efeito de diferentes formas de fornecimento da ração sobre $o$ desempenho e a qualidade da carne de novilhos cruzados em fase de terminação e não verificaram efeito da forma e oferecimento do volumoso sobre o ganho de peso e características da carne.

A utilização da silagem de grão úmido de sorgo é uma prática comum e frequente em sistemas de recria e engorda de bovinos em vários países. Contudo, ainda é escassa informação em relação à possível interação que possa existir entre a forma de fornecimento do volumoso, separado ou misturado, e as características do concentrado, em termos de degradabilidade ruminal, desempenho dos animais, eficiência de conversão alimentar e características de carcaça e carne. Desta forma, objetivou-se verificar o efeito do tipo de silagem sorgo (alto tanino, baixo tanino e branco) e forma de fornecimento da ração (volumoso e concentrado fornecidos de forma separada e misturada) sobre o desempenho e principais características de carcaça de bovinos mestiços de raças britânicas Angus e Hereford terminados em confinamento.

\section{MATERIAL E MÉTODOS}

O estudo foi conduzido no Uruguay com duração de 83 dias. Foram utilizados 36 bovinos machos, castrados, cruzados Hereford-Angus. Os animais foram alocados em 18 baias, dois animais por baia, com um espaço de $11,25 \mathrm{~m}^{2}$ por animal. Os animais foram divididos em blocos casualizados, arranjados em um fatorial $3 \times 2$ (3 tipos de sorgo $\times 2$ formas de fornecimento da ração), considerando 
o peso vivo inicial como bloco. Foram formados três blocos considerando animais com peso vivo inicial baixo, moderado e alto. A unidade experimental foi definida como sendo cada baia.

Os tratamentos foram gerados a partir da combinação de duas formas de fornecimento da ração (volumoso fornecido separado do concentrado ou misturado), e de três tipos de grão úmido de sorgo utilizados (Cultivar IPBFlash 1 com alto teor de tanino, Cultivar S.P.2 com baixo teor de tanino e Cultivar Jowar Food com pericarpo branco), resultando em seis tratamentos: silagem de grão úmido de sorgo contendo alta quantidade de tanino com volumoso separado (SATS); silagem de grão úmido de sorgo contendo alta quantidade de tanino com volumoso misturado (SATM); silagem de grão úmido de sorgo contendo baixa quantidade de tanino com volumoso separado (SBTS); silagem de grão úmido de sorgo contendo baixa quantidade de tanino com volumoso misturado (SBTM); silagem de grão de sorgo úmido com pericarpo branco com volumoso separado (SBRS) e silagem de grão de sorgo com pericarpo branco com volumoso misturado (SBRM).

A composição química e concentração energética das dietas utilizadas para os seis tratamentos é apresentada na Tabela 1. A dieta foi composta por $70 \%$ de grão de sorgo úmido, $10 \%$ de torta de girassol seca e $20 \%$ de feno, com relação de volumoso:concentrado de 20:80, a fim de atender um ganho médio de $1,3 \mathrm{~kg} / \mathrm{dia}$. As exigências nutricionais dos animais, para mantença e ganho de peso, foram ajustadas de acordo com o NRC (2000). $\mathrm{O}$ método para a determinação dos taninos condensados no grão de sorgo foi o método vanilina$\mathrm{HCl}$. Leucoantocianidinas (catequinas) e proantocianidinas (tanino) reagem com a vanilina na presença de $\mathrm{HCl}$ para produzir um produto de condensação vermelho (AGOSTINI-COSTA et al., 2003).

Tabela 1. Composição química e concentração energética das dietas utilizadas

\begin{tabular}{lccc}
\hline $\begin{array}{l}\text { Composição química e } \\
\text { concentração energética }\end{array}$ & $\begin{array}{c}\text { Dieta com sorgo alto } \\
\text { tanino: } \\
\text { SATM e SATS }\end{array}$ & $\begin{array}{c}\text { Dieta com sorgo baixo } \\
\text { tanino: } \\
\text { SBTM e SBTS }^{2}\end{array}$ & $\begin{array}{c}\text { Dieta com sorgo } \\
\text { branco: } \\
\text { SBRS e SBRM }^{3}\end{array}$ \\
\hline Matéria seca, \% & 73 & 75 & 76 \\
Fibra em detergente ácido, \% & 19 & 16 & 17 \\
Fibra em detergente neutro, \% & 35 & 29 & 30 \\
EM, Mcal/Kg MS & 2,45 & 2,50 & 2,50 \\
Cinzas, \% & 4,54 & 4,40 & 4,47 \\
Amônia, \% & 2,1 & 2,7 & 1,4 \\
Potencial hidrogeniônico & 4,6 & 4,2 & 4,3 \\
Tanino & 1,5 & 0,2 & 0,1 \\
Proteína bruta, \% & 12 & 12 & 11 \\
\hline
\end{tabular}

${ }^{1}$ Silagem de grão úmido de sorgo contendo alta quantidade de tanino com volumoso misturado (SATM) e silagem de grão úmido de sorgo contendo alta quantidade de tanino com volumoso separado (SATS).

${ }^{2}$ Silagem de grão úmido de sorgo contendo baixa quantidade de tanino com volumoso misturado (SBTM) e silagem de grão úmido de sorgo contendo baixa quantidade de tanino com volumoso separado (SBTS).

${ }^{3}$ Silagem de grão de sorgo úmido com pericarpo branco com volumoso separado (SBRS) e silagem de grão úmido de sorgo contendo baixa quantidade de tanino com volumoso misturado (SBTM).

Fonte: Laboratório de Nutrição Animal INIA LE 
A matéria seca foi determinada conforme Mieres (2004). A energia metalizável (EM) foi determinada em função da fibra em detergente ácido conforme Rodrigues (2010). As cinzas foram determinadas por incineração da matéria seca a $550{ }^{\circ} \mathrm{C}$ durante três horas e a proteína bruta pelo método Kjeldahl (AOAC, 1984). As fibras em detergente neutro e em detergente ácido determinaram-se pelo método Van Soest (1982). O pH foi determinado pelo método descrito por Silva \& Queiroz (2002). A amônia foi determinada conforme Pigurina (1992).

Foram instalados dois comedouros em cada baia, sendo um para o fornecimento do volumoso e outro para concentrado, para a ração em que o volumoso era fornecido de forma separada do concentrado. Os animais tiveram livre acesso à água, com um bebedouro por baia. A frequência de fornecimento da ração foi de duas vezes ao dia, 9:30 as 10:30 e 16:00 as 17:00 horas. No período da manhã foi fornecida a metade da ração e a outra metade no período da tarde. Os animais tiveram livre acesso aos alimentos durante o dia todo. As sobras foram pesadas diariamente no período da manhã, antes do oferecimento do alimento e a dieta foi ajustada para 5\% da quantidade de matéria seca, fornecida a partir da leitura diária de cocho e a formulação das dietas foi realizada utilizando a metodologia NRC (2000) para ajustar as necessidades dos animais.

A cada 14 dias os animais eram pesados no mesmo horário pela manhã, sem jejum, com o auxílio de uma balança digital (Trutest ID 300®) para a obtenção da evolução do peso vivo, cálculo do ganho de peso médio diário e conversão alimentar (CA=ganho de peso médio diário $(\mathrm{kg} / \mathrm{dia}) /$ consumo médio diário de matéria seca $(\mathrm{kg} / \mathrm{dia}))$. $\mathrm{O}$ ganho de peso médio diário dos animais foi estimado para cada tratamento pela regressão do peso vivo sobre os dias de confinamento utilizando todos os pesos ajustados conforme o modelo proposto na análise estatística para evolução de peso. Desta forma é possível levar em conta a estrutura de correlação entre resíduos que existe entre as medidas de peso para um mesmo animal. A evolução do peso vivo foi obtida em função do peso inicial no confinamento, após 14, 28, 42 e 56 dias de confinamento. A cada período de 28 dias foram realizadas avaliações de ultrassonografia, com a utilização de um equipamento de ultrassom Aloka500D $\AA$, com transdutor de $17 \mathrm{~cm}$ e $3,5 \mathrm{MHz}$, para a determinação de área de olho de lombo (AOLu), espessura de gordura subcutânea (EGSu), marmorização $(\mathrm{MARu})$, no músculo Longissimus, entre a $12^{\mathrm{a}}$ e $13^{\mathrm{a}}$ costelas, do lado direito de cada animal.

Os animais foram abatidos de acordo com o peso vivo final médio de $500 \mathrm{~kg}$, em abatedouro comercial. Após 48 horas de resfriamento, durante a desossa, cada meia carcaça direita foi seccionada entre as $12^{\mathrm{a}}$ e $13^{\mathrm{a}}$ costelas e avaliada a área de olho de lombo (AOL, $\mathrm{cm}^{2}$ ), espessura de gordura subcutânea (EGS, mm) com o auxílio de grid específico. Também foi mensurado o pH (às 48 horas e aos 14 dias), com pHmetro portátil Hanna instruments e a MAR, utilizando-se um padrão USDA (QUALITY GRADE, 1999). Foram retiradas amostras do músculo Longissimus entre a $12^{\mathrm{a}}$ e $13^{\mathrm{a}}$ costelas, para avaliação do $\mathrm{pH}$, cor ( $\mathrm{L}^{*}, \mathrm{a}^{*}$ e $\left.\mathrm{b}^{*}\right)$ e força de cisalhamento (FC, kgf). Os cortes foram identificados, embalados a vácuo e maturados a $2^{\circ} \mathrm{C}$, durante 14 dias, e em seguida congelados $-30^{\circ} \mathrm{C}$, para posteriores análises descritas a seguir. 
A análise de cor foi realizada, com o auxílio de um colorímetro Minolta (C10), seguindo a escala CIE Lab, para os valores de $L^{*}, a^{*} e b^{*}$. A maciez foi analisada com equipamento Warner Bratzler (modelo D2000), como a média de 6 cilindros de cada amostra, segundo o protocolo AMSA (1995).

Foram analisadas as características de consumo absoluto diário de matéria seca (CMSa), consumo relativo de matéria seca (CMSr), expressos como percentagem do peso vivo, ganho de peso médio diário (GMD), conversão alimentar (CA), MARu, AOLu, EGSu, AOL, EGS, MAR, peso do serrote, contrafilé, alcatra e filé mignon, $\mathrm{pH}$ após 48 horas do abate e 14 dias e maciez objetiva. Para análise da conversão alimentar, características de carcaça (AOL, EGS e MAR, post mortem), peso de cortes cárneos (serrote, contra filé, alcatra e filé mignon), cor, maciez, $\mathrm{pH}$ (com 48 horas e 14 dias), utilizou-se o procedimento MIXED do SAS 9.3. (SAS Institute, Cary, NC, USA), aplicando a metodologia de máxima verossimilhança restrita, sendo o modelo utilizado.

$\mathrm{Y}_{\mathrm{ijk}}=\mu+\beta_{\mathrm{i}}+\alpha_{\mathrm{j}}+\gamma_{\mathrm{k}}+(\alpha \gamma)_{\mathrm{jk}}+\varepsilon_{\mathrm{ijk}}$

Onde, $Y_{\mathrm{ijk}}$ característica avaliada; $\mu$ : média geral; $\beta_{\mathrm{i}}$ : efeito aleatório do iésimo bloco ( $i=1,2,3$ ou 4); $\alpha_{j}$ : efeito fixo do j-ésimo tipo de sorgo $(\mathrm{j}=1,2$ ou 3); $\gamma_{\mathrm{k}}$ : efeito fixo da k-ésima forma de fornecimento da ração $(\mathrm{k}=1$ ou 2$)$; $(\alpha \gamma)_{\mathrm{jk}}$ : efeito fixo da interação entre o jésimo tipo de sorgo com a k-ésima forma de fornecimento da ração; $\varepsilon_{\mathrm{ijk}}$ : erro experimental.

Para as características de CMSa, CMSr, evolução do peso vivo, ganho de peso médio diário, características de carcaça avaliadas in vivo (AOLu, EGSu e MARu), foi utilizada uma análise de medidas repetidas no tempo, com o procedimento MIXED do SAS (SAS 9.3, SAS Institute, Cary, NC, USA), aplicando a metodologia de máxima verossimilhança restrita, sendo o modelo utilizado:

$\mathrm{Y}_{\mathrm{ijkl}}=\mu+\beta_{\mathrm{i}}+\alpha_{\mathrm{j}}+\gamma_{\mathrm{k}}+(\alpha \gamma)_{\mathrm{jk}}+\delta_{\mathrm{ijk}}+\tau_{\mathrm{l}}$

$+(\alpha \tau)_{\mathrm{jl}}+(\gamma \tau)_{\mathrm{lk}}+(\alpha \gamma \tau)_{\mathrm{jkl}}+\varepsilon_{\mathrm{ijkl}}$

Onde, $\mathrm{Y}_{\mathrm{ijkl}}$ : característica avaliada; $\mu$ : média geral; $\beta_{\mathrm{i}}$ : efeito aleatório do $\mathrm{i}$ ésimo bloco ( $i=1,2,3$ ou 4$) ; \alpha_{j}$ : efeito fixo do j-ésimo tipo de sorgo $(\mathrm{j}=1,2$ ou 3); $\gamma_{k}$ : efeito fixo da k-ésima forma de fornecimento da ração $(\mathrm{k}=1$ ou 2$)$; $(\alpha \gamma) \mathrm{j}_{\mathrm{k}}$ : efeito da interação entre $\mathrm{o} \mathrm{j}$ ésimo tipo sorgo com a k-ésima forma de fornecimento da ração; $\delta_{\mathrm{ijk}}$ : erro experimental; $\tau_{1}$ : efeito do l-ésimo período; $(\alpha \tau)_{\mathrm{j} 1}$ : efeito da interação entre o j-ésimo tipo de sorgo e o l-ésimo período; $(\gamma \tau)_{\mathrm{lk}}$ : efeito da interação entre a k-ésima forma de fornecimento da ração e o 1-ésimo período; $(\alpha \gamma \tau)_{\mathrm{jkl}}$ : efeito da interação entre o j-ésimo tipo de sorgo, k-ésima forma de fornecimento da ração e l-ésimo período; $\varepsilon_{\mathrm{ijk}}$ : erro experimental.

$\mathrm{O}$ ganho de peso médio diário de cada tratamento foi comparado utilizando-se contrastes ortogonais. Para todas as análises utilizou-se o programa $\mathrm{SAS}$ (SAS 9.3, SAS Institute, Cary, NC, USA). Nas análises de variância, quando os efeitos foram significativos $(\mathrm{p}<0,05)$ utilizou-se o Teste de TukeyKramer para a comparação de médias.

\section{RESULTADOS E DISCUSSÃO}

Para a característica consumo de matéria seca absoluto (CMSa) e evolução do peso vivo houve interação $(\mathrm{P}=0,0001)$ entre o tipo de sorgo e forma de fornecimento da ração (Tabela 2). Os animais alimentados com ração contendo sorgo com alto teor de tanino e volumoso separado apresentaram maior $\mathrm{CMSa}(\mathrm{P}=0,0001)$. Entretanto, os animais alimentados com sorgo com 
Rev. Bras. Saúde Prod. Anim., Salvador, v.17, n.4, p.685-695 out./dez., 2016 http://www.rbspa.ufba.br ISSN 15199940

baixo tanino e o volumoso separado e os alimentados com a dieta contendo sorgo branco e volumoso misturado foram os que apresentaram o menor CMSa $(\mathrm{P}=0,0001)$. Os tratamentos SATS e SBTS não apresentaram diferenças em peso corporal médio, em relação àqueles que receberam as dietas SATM e SBTM, respectivamente
$(\mathrm{P}=0,3967$ e $\mathrm{P}=0,1991)$. Entretanto, os animais que consumiram a dieta SBRS apresentaram maior peso corporal, quando comparados aos que receberam SBRM $(\mathrm{P}=0,0020)$. Portanto, o tipo de silagem de grão de sorgo e a forma de fornecimento da ração afetou a evolução de peso dos animais ao longo do período experimental.

Tabela 2. Médias ajustadas e erro padrão da média para as características consumo diário de matéria seca absoluto (CMSa) e peso vivo médio (PVM) de acordo com o tipo de sorgo e forma de fornecimento da ração, e respectivo valor de probabilidade $(\mathrm{P})$

\begin{tabular}{lcc}
\hline \multicolumn{1}{c}{ Tratamento } & CMSa, kg MS/animal/dia & PVM, kg \\
\hline SATS & $22,83 \pm 0,78^{\mathrm{a}}$ & $414,5 \pm 9,87^{\mathrm{bcd}}$ \\
SATM & $20,78 \pm 0,78^{\mathrm{c}}$ & $402,5 \pm 9,87^{\mathrm{ab}}$ \\
\hline SBTS & $19,24 \pm 0,78^{\mathrm{d}}$ & $409,7 \pm 9,87^{\mathrm{abcd}}$ \\
SBTM & $20,98 \pm 0,78^{\mathrm{c}}$ & $424,1 \pm 9,87^{\mathrm{d}}$ \\
\hline SBRS & $22,37 \pm 0,78^{\mathrm{b}}$ & $420,6 \pm 9,87^{\mathrm{cd}}$ \\
SBRM & $19,37 \pm 0,78^{\mathrm{d}}$ & $396,3 \pm 9,87^{\mathrm{a}}$ \\
\hline P & 0,0001 & 0,0001 \\
\hline
\end{tabular}

SATS = silagem de grão úmido de sorgo contendo alta quantidade de tanino com volumoso separado.

SATM = silagem de grão úmido de sorgo contendo alta quantidade de tanino, com volumoso misturado. SBTS = silagem de grão úmido de sorgo contendo baixa quantidade de tanino com volumoso separado.

SBTM = silagem de grão úmido de sorgo contendo baixa quantidade de tanino, com volumoso misturado.

SBRS = silagem de grão de sorgo úmido com pericarpo branco com volumoso separado.

Para as características conversão alimentar (CA), consumo de matéria seca relativo (CMSr), área de olho de lombo (AOLu e AOL), marmorização (MARu e MAR) e espessura de gordura dorsal (EGSu e EGS) por ultrassonografia e na desossa respectivamente, não houve efeito de interação entre tipo de silagem de sorgo e forma de fornecimento do volumoso $(\mathrm{p}>0,05)$. Para o $\mathrm{CMSr}$, houve efeito do tipo de sorgo $(\mathrm{P}=0,002)$ e forma da fornecimento da ração $(\mathrm{P}=0,0005)$, destacando-se um menor consumo relativo como percentagem do peso vivo no grupo de animais que receberam dieta contendo grão de sorgo com baixo tanino, independente da forma de fornecimento da ração. Os animais alimentados com volumoso separado do concentrado, independentemente do tipo de grão, tiveram um maior $\mathrm{CMSr}(\mathrm{P}=0,0005)$ em relação àqueles que receberam o volumoso misturado com concentrado (Tabela 3) .

No que diz respeito à conversão alimentar, não foi observado efeito da forma de fornecimento da ração $(\mathrm{P}=0,30)$. Entretanto, observou-se efeito do tipo de grão de sorgo $(\mathrm{P}=0,04)$ sobre a conversão alimentar. Os bovinos que receberam silagem de sorgo com alto tanino tiveram maior conversão alimentar, enquanto que os animais que receberam silagem de sorgo com baixo teor de tanino tiveram menor conversão alimentar (Tabela 3). A melhor conversão alimentar da silagem 
Rev. Bras. Saúde Prod. Anim., Salvador, v.17, n.4, p.685-695 out./dez., 2016 http://www.rbspa.ufba.br

de sorgo de baixo teor em tanino pode ser explicada pela maior degradação em nível ruminal da matéria seca, quando comparado a silagem de sorgo com alto teor de tanino. Estes resultados também foram observados por Cação et al. (2012), que verificaram maior degradação ruminal em silagem de grão de sorgo com baixo teor de tanino, em comparação a silagem de grão de sorgo com alto teor de tanino.

Para as características AOLu e EGSu não houve efeito do tipo de sorgo utilizado $(\mathrm{P}=0,051 ; \mathrm{P}=0,54)$ nem da forma de fornecimento da ração $(\mathrm{P}=0,557 ; \mathrm{P}=0,75)$.
Para a MARu, houve efeito do tipo de sorgo $(\mathrm{P}=0,001)$, com maior teor de marmorização nas carnes de animais que consumiram dieta contendo sorgo com pericarpo branco; entretanto, não houve efeito da forma de fornecimento da ração $(\mathrm{P}=0,65)$ para esta característica. Não foi observado efeito do tipo de sorgo $(\mathrm{P}=0,09 ; \mathrm{P}=0,15$ e $\mathrm{P}=0,89)$ para $\mathrm{AOL}$, MAR e EGS, respectivamente; assim como efeito da forma de fornecimento da ração $(\mathrm{P}=0,13 ; \mathrm{P}=0,25)$ para a $\mathrm{AOL} \mathrm{e}$ EGS respectivamente, entretanto esse efeito foi observado $(\mathrm{P}=0,04)$ para MAR (Tabela 3).

Tabela 3. Médias ajustadas e erro padrão da média para a característica conversão alimentar (CA) e consumo de matéria seca relativo (CMSr), área de olho de lombo (AOLu e AOL), marmorização (MARu e MAR) e espessura de gordura dorsal (EGSu e EGS) por ultrassonografia e na desossa respectivamente, em função do tipo de grão de sorgo e da forma de fornecimento da ração (FFR)

\begin{tabular}{|c|c|c|c|c|c|c|c|}
\hline \multirow{2}{*}{ Característica } & \multicolumn{3}{|c|}{ Tipo de sorgo } & \multirow{2}{*}{$\mathrm{P}$} & \multicolumn{2}{|c|}{ FFR } & \multirow{2}{*}{$\mathrm{P}$} \\
\hline & Branco & Baixo Tanino & Alto Tanino & & Misturado & Separado & \\
\hline $\mathrm{CMSr}, \% \mathrm{PV}$ & $2,47 \pm 0,40^{\mathrm{ab}}$ & $2,38 \pm 0,40^{\mathrm{b}}$ & $2,55 \pm 0,40^{\mathrm{a}}$ & 0,002 & $2,40 \pm 0,36^{\mathrm{b}}$ & $2,53 \pm 0,36^{\mathrm{a}}$ & 0,0005 \\
\hline CA, kg MS/ kg PV & $7,41 \pm 0,29^{\mathrm{ab}}$ & $6,80 \pm 0,28^{\mathrm{b}}$ & $7,98 \pm 0,31^{\mathrm{a}}$ & 0,04 & $7,58 \pm 0,24$ & $7,22 \pm 0,24$ & 0,30 \\
\hline $\mathrm{AOLu}\left(\mathrm{cm}^{2}\right)$ & $64,86 \pm 0,87$ & $65,88 \pm 0,88$ & $63,64 \pm 0,90$ & 0,08 & $65,17 \pm 0,82$ & $64,42 \pm 0,84$ & 0,58 \\
\hline MARu (\%) & $3,22 \pm 0,05^{\mathrm{a}}$ & $2,81 \pm 0,05^{\mathrm{b}}$ & $2,68 \pm 0,05^{\mathrm{b}}$ & 0,001 & $2,89 \pm 0,04$ & $2,92 \pm 0,04$ & 0,65 \\
\hline $\mathrm{EGSu}(\mathrm{mm})$ & $5,00 \pm 0,20$ & $4,89 \pm 0,21$ & $4,75 \pm 0,21$ & 0,54 & $4,85 \pm 0,18$ & $4,91 \pm 0,18$ & 0,75 \\
\hline $\operatorname{AOL}\left(\mathrm{cm}^{2}\right)$ & $61,66 \pm 1,39$ & $61,40 \pm 1,39$ & $57,61 \pm 1,39$ & 0,09 & $61,49 \pm 1,114$ & $58,90 \pm 1,14$ & 0,13 \\
\hline MAR (\%) & $6,18 \pm 0,36$ & $5,52 \pm 0,36$ & $5,48 \pm 0,36$ & 0,15 & $5,46 \pm 0,29^{b}$ & $6,66 \pm 0,29^{\mathrm{a}}$ & 0,04 \\
\hline EGS (mm) & $11,47 \pm 0,38$ & $11,73 \pm 0,38$ & $11,64 \pm 0,38$ & 0,89 & $11,83 \pm 0,31$ & $11,35 \pm 0,81$ & 0,25 \\
\hline
\end{tabular}

Observou-se um maior consumo absoluto dos animais que ingeriram volumoso separado do concentrado, nas dietas contendo sorgo branco ou de alto tanino, coincidindo com dados relatados por Collares et al. (2008). No entanto, Atwood et al. (2001) verificaram um maior consumo em bovinos, ao utilizar a ração com volumoso e concentrado misturado. Por outro lado, La Manna et al. (2012) não observaram efeito da forma de fornecimento da ração sobre $\mathrm{o}$ consumo dos animais.

No presente trabalho não foi observado o efeito depressor dos taninos sobre $\mathrm{o}$ consumo de matéria seca. Também, Maxson et al. (1973) e Riffel (2002) verificaram maior consumo de matéria seca em ração contendo alto teor de tanino. Provavelmente, houve degradação dos taninos do grão durante o processo de ensilagem, reduzindo a concentração 
deste fator anti-nutricional, sem acarretar redução do consumo de matéria seca nos animais alimentados com ração de alto teor em tanino (BHATET al., 1998). Neste sentido, Cação et al. (2012) relataram um decréscimo nos teores de fenóis totais, tanino total e tanino condensado em grãos de sorgo com alto e baixo conteúdo de tanino, quando os mesmos foram ensilados.

As diferenças encontradas no consumo de matéria seca entre os tratamentos não foram devido às variações nas concentrações energéticas das dietas, uma vez que a mesma foi semelhante. A alta quantidade de tanino presente no grão de sorgo pode ter reduzido a degradação ruminal da dieta, estimulando um maior consumo de matéria seca relativo $(\mathrm{CMSr})$ pelos animais, devido a possível redução da saciedade dos animais. De acordo com os resultados obtidos no presente estudo, a estratégia utilizada para o fornecimento da ração influencia o consumo de matéria seca dos animais, sendo que os animais que receberam volumoso separado do concentrado apresentaram maior CMSr e maior (CMSa), quando foi utilizado sorgo de alto e baixo teor de tanino.

Para o ganho de peso médio diário, houve interação entre o tipo de sorgo e forma de fornecimento da ração, sendo que, os animais que receberam silagem de grão de sorgo de baixo teor de tanino com volumoso separado apresentaram maior ganho de peso médio diário $(\mathrm{P}=0,003) \mathrm{em}$ comparação aos animais alimentados com o mesmo tipo de silagem de grão de sorgo, mas com o volumoso misturado (Tabela 4). Em trabalhos que utilizaram diferentes formas de fornecimento de ração, como as utilizadas no presente estudo, não foram observadas diferenças sobre o ganho de peso médio diário (ATWOOD et al., 2001; COLLARES et al., 2008; La MANNA et al., 2012). De acordo com McSweeney et al. (2001), o tanino pode reduzir a digestão da fibra, podendo diminuir o consumo de matéria seca. Como foi utilizada baixa quantidade de fibra neste estudo, não era esperado que este fator tivesse efeito sobre o desempenho animal. Portanto, há indicações de que a estratégia utilizada para a forma de fornecimento da ração e o tipo de grão de sorgo influencia a evolução de peso vivo e o ganho de peso dos animais. Há vantagem do ponto de vista operacional e nutricional para animais que receberam o volumoso separado do concentrado, uma vez que a resposta produtiva foi maior quando o volumoso é fornecido separado do concentrado. Além disso, é dispensável a necessidade de um vagão misturador com a consequente redução dos custos, tempo de alimentação dos animais, funcionários para operar máquinas e infraestrutura.

Em relação aos pesos dos cortes cárneos, cor, maciez e pH não foi observado efeito significativo da interação entre tipo de grão de sorgo com forma de fornecimento da ração $(p>0,05)$, tampouco efeito do tipo de grão de sorgo $(\mathrm{p}>0,05)$ e da forma de fornecimento da ração $(p>0,05)$ (Tabela 5). Estes resultados corroboram com os encontrados por Pordomingo et al. (2004), que não observaram efeito do teor de tanino sobre a maciez nem sobre a cor dos cortes cárneos de bovinos.

A ausência de efeito da forma de fornecimento da ração sobre as características de carcaça também foi relatada por La Manna et al. (2012) e Collares et al. (2008). Provavelmente, a diferença em ganho de peso médio diário entre os animais que receberam concentrado e volumoso de forma separada ou misturada não foi suficientemente significativa para justificar alterações nas características de carcaça e carne. A ausência de diferença para o efeito do tipo de grão de sorgo nos resultados encontrados para o peso dos diferentes cortes cárneos também foi observada por Maxon et al. (1973). 
Rev. Bras. Saúde Prod. Anim., Salvador, v.17, n.4, p.685-695 out./dez., 2016 http://www.rbspa.ufba.br ISSN 15199940

Tabela 4. Média e erro padrão da média $(\mu \pm$ se) das características de ganho de peso médio diário (GMD), em função da interação entre o tipo de grão de sorgo (grão úmido de sorgo com alto teor de tanino -SAT, grão úmido de sorgo com baixo teor de tanino SBT e grão de sorgo úmido com pericarpo branco SBR) e forma de fornecimento da ração (concentrado e volumoso misturado - MIST ou separado - SEP), seguidas pelos valores de F e p

\begin{tabular}{|c|c|c|c|c|}
\hline \multirow{2}{*}{ Contraste } & \multicolumn{2}{|c|}{ GMD, kg } & \multirow{2}{*}{$\mathrm{F}$} & \multirow{2}{*}{$\mathrm{P}$} \\
\hline & $\mu \pm \mathrm{se}$ & $\mu \pm \mathrm{se}$ & & \\
\hline MIST vs SEP & $1,310 \pm 0,099$ & $1,535 \pm 0,099$ & 0,23 & 0,629 \\
\hline SAT vs SBT & $1,302 \pm 0,122$ & $1,457 \pm 0,122$ & 0,85 & 0,357 \\
\hline SBR vs SBT & $1,509 \pm 0,122$ & $1,457 \pm 0,122$ & 0,10 & 0,754 \\
\hline SAT vs SBR & $1,302 \pm 0,122$ & $1,509 \pm 0,122$ & 1,53 & 0,218 \\
\hline SATM vs SATS & $1,163 \pm 0,145$ & $1,442 \pm 0,145$ & 2,72 & 0,101 \\
\hline SBRM vs SBRS & $1,564 \pm 0,145$ & $1,453 \pm 0,145$ & 0,44 & 0,510 \\
\hline SBTM vs SBTS & $1,202 \pm 0,145$ & $1,711 \pm 0,145$ & 9,02 & 0,003 \\
\hline
\end{tabular}

SATS = silagem de grão úmido de sorgo com alto teor de tanino com volumoso separado.

SATM = silagem de grão úmido de sorgo com alto teor de tanino com o volumoso misturado.

SBTM = silagem de grão úmido de sorgo com baixo teor de tanino com o volumoso misturado.

SBRS = silagem de grão de sorgo úmido com pericarpo branco com volumoso separado.

SBRS = silagem de grão de sorgo úmido com pericarpo branco com volumoso separado.

$\mathrm{SBRM}=$ silagem de grão de sorgo com pericarpo branco, com volumoso misturado .

Tabela 5. Médias e erro padrão para os diferentes cortes cárneos (contra filé, alcatra, filé mignon e traseiro), maciez, cor (luminosidade $L^{*}$ e cromância $a^{*}$ e b*), $\mathrm{pH}$ (às 48 horas e aos 14 dias), em função do tipo de grão de sorgo e da forma de fornecimento da ração (FFR)

\begin{tabular}{|c|c|c|c|c|c|c|c|}
\hline \multirow{2}{*}{ Características } & \multicolumn{3}{|c|}{ Tipo de sorgo } & \multirow{2}{*}{$\mathrm{P}$} & \multicolumn{2}{|c|}{ FFR } & \multirow{2}{*}{$P$} \\
\hline & Branco & Baixo Tanino & Alto Tanino & & Misturado & Separado & \\
\hline Contra filé (kg) & $5,69 \pm 1,17$ & $5,89 \pm 1,17$ & $5,56 \pm 1,17$ & 0,35 & $5,66 \pm 0,15$ & $5,77 \pm 0,15$ & 0,59 \\
\hline Alcatra (kg) & $3,68 \pm 0,10$ & $3,73 \pm 0,10$ & $3,70 \pm 0,10$ & 0,92 & $3,75 \pm 0,09$ & $3,67 \pm 0,09$ & 0,44 \\
\hline Filé Mignon (kg) & $2,45 \pm 0,07$ & $2,48 \pm 0,07$ & $2,38 \pm 0,07$ & 0,11 & $2,46 \pm 0,06$ & $2,40 \pm 0,06$ & 0,12 \\
\hline Traseiro (kg) & $58,66 \pm 1,67$ & $58,58 \pm 1,67$ & $56,49 \pm 1,67$ & 0,29 & $57,84 \pm 1,55$ & $57,98 \pm 1,55$ & 0,92 \\
\hline Maciez & $3,89 \pm 0,23$ & $3,89 \pm 0,23$ & $3,73 \pm 0,23$ & 0,86 & $3,94 \pm 0,18$ & $3,73 \pm 0,18$ & 0,44 \\
\hline Cor $\mathrm{L}^{*}$ & $35,16 \pm 0,74$ & $34,84 \pm 0,74$ & $34,30 \pm 0,74$ & 0,72 & $34,89 \pm 0,61$ & $34,65 \pm 0,61$ & 0,78 \\
\hline Cor $a^{*}$ & $15,96 \pm 0,54$ & $14,03 \pm 0,54$ & $15,47 \pm 0,54$ & 0,06 & $15,59 \pm 0,44$ & $14,71 \pm 0,44$ & 0,18 \\
\hline Cor $b^{*}$ & $7,93 \pm 0,20$ & $7,48 \pm 0,20$ & $7,90 \pm 0,20$ & 0,19 & $7,72 \pm 0,18$ & $7,81 \pm 0,18$ & 0,69 \\
\hline pH às 48hs & $5,34 \pm 0,40$ & $5,38 \pm 0,40$ & $5,45 \pm 0,40$ & 0,13 & $5,37 \pm 0,33$ & $5,42 \pm 0,33$ & 0,69 \\
\hline $\mathrm{pH}$ aos 14 dias & $5,72 \pm 0,13$ & $5,68 \pm 0,13$ & $5,72 \pm 0,13$ & 0,40 & $5,69 \pm 0,41$ & $5,72 \pm 0,41$ & 0,27 \\
\hline
\end{tabular}

Dependendo do tipo de grão de sorgo utilizado, a forma de fornecimento da ração pode interferir sobre o consumo de matéria seca e ganho de peso dos novilhos. $\mathrm{O}$ fornecimento de concentrado e volumoso de forma separada para bovinos em confinamento, do ponto de vista operacional e econômico é viável. A presença de tanino na silagem de grão úmido de sorgo afetou a eficiência de conversão alimentar dos novilhos. O tipo de grão sorgo e a forma de fornecimento 
Rev. Bras. Saúde Prod. Anim., Salvador, v.17, n.4, p.685-695 out./dez., 2016 http://www.rbspa.ufba.br ISSN 15199940

da ração não influenciam as principais características de carcaça e pesos dos cortes cárneos considerados. Os resultados deste trabalho poderão auxiliar e fornecer suporte para sistemas de engorda de bovinos em confinamento que utilizam silagem de grão úmido de sorgo e não possuem maquinaria (mixer) para a elaboração de raçoes totalmente misturada.

\section{REFERÊNCIAS}

AGOSTINI-COSTA, T.S; LIMA, A.; LIMA, M.V. Determinação de tanino em pedúnculo de caju: método da vanilina versus método do butanol ácido. Química Nova, v.6, p.763-765, 2003.

AMERICAN MEAT SCIENCE ASSOCIATION - AMSA. Research guidelines forcookery, sensory evaluation, and instrumental tenderness measurements of fresh meat. Chicago, 1995. 47p.

ASSOCIATION OF OFFICIAL AGRICULTURAL CHEMISTS AOAC. Official methods of analysis.14th ed. Washington, D.C, 1984.

ATWOOD, S.B.; PROVENZA, F.D.; WIEDMEIER, R.D.; BANNER, R.E. Influence of free-choice vs mixed-ration diets on food intake and performance of fattening calves. Journal of Animal Science, v.79, n.12, p.3034-3040, 2001.

BHAT, T.K.; SINGH, B.; SHARMA, O.P. Microbial degradation of tanins: A current perspective. Biodegradation, v.9, p.343-357, 1998.
CAÇÃO, M.M.D.F.; COSTA, C.; MEIRELLES, P.R.D.L.; EZEQUIEL, J.M.B.; GALATI, R.L.; SILVA, M.G.B. Degradabilidaderuminal da matéria seca de grãos de milho de sorgo com alto ou baixo conteúdo de tanino processados.

Revista Brasileira de Saúde e Produção Animal [online], v.13, n.2, p.516-528, 2012.

COLLARES, M. Manejo de la fibra en sistemas de alimentación a corral para vacunos en crecimiento $y$ terminación. TesisIng. Agr.

Montevideo, Uruguay: Facultad de Agronomía, 2008. 77p.

GARCIA-DE-SILES, J. L.; ZIEGLER, J. H.; WILSON, L. L.Effects of marbling and conformation scores on quality and quantity characteristics of steer and heifer carcasses. Journal of Animal Science, v.44, n.1, p.36-46, 1977.

JENNINGS, T.G.; BERRY, B.W.; JOSEPH, A.L. Influence of fat thickness, marbling and length of aging on beef palatability and shelf-life characteristics. Journal of Animal Science, v.46, n.3, p.658-665, 1978.

La MANNA, A. F.; BANCHERO G.E.; FERNÁNDEZ E.; BARBOSA J.D.; HERRERA A.; PATRONE J.P.

Frecuencia de suministro y presentación de la dieta sobre el comportamiento productivo de novillos en engorde a corral. In: IV CONGRESSO DEL ASSOCIACIÓN URUGUAYA DE PRODUCIÓN ANIMAL, 29 a 30 de Octubro 2012, Anais... Montevideo, Sociedad de Medicina Veterinaria del Uruguay, 2012, v.48, p.148.

Disponívelem:

$<$ http://www.revistasmvu.com.uy/revist as/congreso-aupa.pdf $>$. Acesso em: 25 Abr. 2014. 
Rev. Bras. Saúde Prod. Anim., Salvador, v.17, n.4, p.685-695 out./dez., 2016 http://www.rbspa.ufba.br ISSN 15199940

MAXSON, W.E.; SHIRLEY, R.L.; BERTRAND, J.E.; PALMER, A.Z. Energy values of corn, bird-resistant and non-bird-resistant sorghum grain in rations fed to steers. Journal of Animal Science, v.37, p.1451-1457, 1973.

MCSWEENEY, C.S.; PALMER, B.; MCNEILL, D.M.; KRAUSE, D.O. Microbial interactions with tannins: nutritional consequences for ruminants. Animal Feed Science and Technology, v.91, n.1, p.83-93, 2001.

MIERES, J. M. Guía para la alimentación de rumiantes. Colonia, Uruguay: INIA, 2004. (Serie Técnica).

NATIONAL RESEARCH COUNCIL NRC. Nutrient requirements of beef cattle. Washington, D.C.: National Academic Press, 2000.

PIGURINA, G. Características de ensilajes en establecimientos lecheros. Colonia, Uruguay: INIA La Estanzuela, 1992.

PORDOMINGO, A.J.; VOLPI

LAGRECA, G.; PILAR, G.;

GRIGIONI, G. Efecto del agregado de taninos en dietas de distinto nivel de grano en vaquillonas para carne alimentadas en confinamiento sobre la calidad de la carne. Boletín Técnico, v.88, cap.14, p.72-82, 2004.

RIFFEL, S. Estudio comparativo entre el "grano de maíz" y el "grano de sorgo con bajo y alto contenido de taninos". INTA EEA. Bordenave CC Nro. 44 - 8187. Buenos Aires, Argentina, 2002.

RODRIGUES, R.C. Métodos de análises bromatológicas de alimentos: métodos físicos, químicos e bromatológicos. Pelotas, RS: Embrapa Clima Temperado, 2010. (Documentos).
ROONEY, L.W.; PFLUGFELDER, R.L. Factors affecting starch digestibility with special emphasis on sorghum and corn. Journal of Animal Science, v.63, n.5, p.1607-1623, 1986.

SILVA, D.J.; QUEIROZ, A.C. Análise de alimentos: métodos químicos e biológicos. 3.ed. Viçosa, MG: UFV, 2002.

TEIRA, G. Actualidad y perspectivas de un componente principal de la calidad de carnes bovinas: la terneza. Ciencia, Docencia y Tecnología, v.15, n.28, p.215-244, 2004.

TUMA, H.J.; HENRICKSON, R.L.; STEPHENS, D.F.; MOORE, R. Influence of marbling and animal age on factors associated with beef quality. Journal of Animal Science, v.21, n.4, p.848-851, 1962.

\section{USDA. Official United States} standards for grades of carcass beef. Agric. Marketting Serv., USDA, Washington, D.C., 1999.

VAN SOEST, P.J. Nutritional Ecology of Ruminant. New York: Cornell University Press, 1982.

Data de recebimento: 19/03/2015 Data de aprovação: 31/08/2016 